Márcia Inês Boff', Márcio Pereira Hetzel$^{2}$, Daniele Munaretto Dallegrave ${ }^{2}$, Roselaine Pinheiro de Oliveira $^{2}$, Claúdia da Rocha Cabral ${ }^{3}$, Cassiano Teixeira ${ }^{2,4}$

1. Médica do Complexo Hospitalar da Santa Casa de Porto Alegre - Porto Alegre (RS), Brasil.

2. Médicos da Unidade de Terapia Intensiva Central do Complexo Hospitalar da Santa Casa de Porto Alegre - Porto Alegre (RS), Brasil. 3. Enfermeira, pós-graduanda do curso de Enfermagem Curso de Enfermagem em Terapia Intensiva da Universidade do Vale do Rio dos Sinos - UNISINOS Porto Alegre (RS), Brasil.

4. Professor Adjunto da Universidade Federal de Ciências da Saúde de Porto Alegre - UFSCPA - Porto Alegre (RS), Brasil.

Estudo realizado na Unidade de Terapia Intensiva Central do Complexo Hospitalar da Santa Casa de Porto Alegre - Porto Alegre (RS), Brasil.

Os autores não têm conflito de interesse na realização ou publicação do estudo.

Submetido em 14 de Dezembro de 2008

Aceito em 24 de Novembro de 2009

Autor para correspondência:

Cassiano Teixeira

Rua Riveira, 355 / 403

CEP: 90670-160 - Porto Alegre (RS),

Brasil.

E-mail: cassiano.rush@terra.com.br

\section{Perfil e prognóstico a longo prazo dos pacientes que recebem terapia insulínica em unidades de terapia intensiva clínico-cirúrgica: estudo de coorte}

\author{
Profile and long-term prognosis of glucose tight control in \\ intensive care unit-patients: a cohort study
}

\section{RESUMO}

Objetivos: Hiperglicemia induzida por estresse ocorre com freqüência em pacientes criticamente doentes e tem sido associada a aumento de mortalidade e morbidade tanto em pacientes diabéticos, quanto em não diabéticos. $\mathrm{O}$ objetivo deste estudo foi avaliar o perfil e prognóstico a longo prazo dos pacientes críticos que recebem terapia insulínica contínua na unidade de terapia intensiva.

Métodos: Coorte prospectiva, em que foram estudados os pacientes internados na unidade de terapia intensiva no período de 1 ano. Foram analisadas variáveis demográficas, escores de gravidade e o prognóstico a curto na unidade de terapia intensiva, e a longo prazo ( 2 anos da alta da unidade de terapia intensiva). Os pacientes foram classificados em 2 grupos: pacientes que receberam terapia insulínica contínua para controle glicêmico indicada pela equipe da unidade de terapia intensiva e pacientes que não receberam terapia insulínica.

Resultados: Dos 603 pacientes incluídos no estudo, $102(16,9 \%)$ receberam terapia insulínica contínua, objetivando níveis glicêmicos $<150 \mathrm{mg} / \mathrm{dL}$ e 501 pacientes
$(83,1 \%)$ não receberam insulina contínua. Os pacientes que necessitaram terapia insulínica contínua eram mais graves que os do grupo não necessitou de terapia insulínica: escore APACHE II (14 \pm 3 versus $11 \pm 4$; $\mathrm{p}$ $=0,04)$, escore SOFA $(4,9 \pm 3,2$ versus 3,5 $\pm 3,4 ; \mathrm{p}<0,001)$ e TISS das $24 \mathrm{~h}(25,7 \pm 6,9$ versus $21,1 \pm 7,2 ; \mathrm{p}<0,001)$. Os pacientes do grupo que recebeu terapia insulínica contínua tiveram também pior prognóstico: insuficiência renal aguda $(51 \%$ versus $18,5 \%$; $\mathrm{p}<0,001)$, polineuropatia da doença crítica $(16,7 \%$ versus $5,6 \% ; p<0,001)]$ e maior mortalidade [na unidade de terapia intensiva $(60,7 \%$ versus $17,7 \%$; $p<0,001)$ e 2 anos após a alta da unidade de terapia intensiva ( $77,5 \%$ versus $23,4 \%$; $\mathrm{p}<0,001$ ).

Conclusão: A necessidade de controle glicêmico rigoroso através do uso de protocolos de insulina contínua é um marcador de gravidade e de pior prognóstico dos pacientes internados na unidade de terapia intensiva, refletindo também maior mortalidade a longo prazo.

Descritores: Glicemia/análise; Hiperglicemia/prevenção \& controle; Insulina/ uso terapêutico; Hipoglicêmicos/uso terapêutico; Prognóstico; Cuidados intensivos

\section{INTRODUÇÃO}

A hiperglicemia induzida por estresse ocorre com freqüência em pacientes internados em unidade de tratamento intensivo (UTI) e tem sido associada ao aumento de mortalidade e morbidade em pacientes diabéticos e não diabéticos. ${ }^{(1)}$ Os mecanismos pelos quais a hiperglicemia piora o desfecho nesses pacientes podem ser relacionados a efeitos supressivos na funçáo imunológica e ao aumento associado no risco de infecção, disfunção endotelial, injúria mitocondrial hepatocitária e a possível isquemia tecidual devido à acidose ou inflamaçẫo. ${ }^{(2)}$ 
A hiperglicemia predispõe a distúrbios do sódio, potássio e fósforo. ${ }^{(3)}$ A diurese osmótica, secundária a hiperosmolaridade sérica, pode gerar hiponatremia sintomática. A hipocalemia aumenta a chance de arritmias e a hipofosfatemia pode interferir na função plaquetária e leucocitária. Alguns microorganismos podem tornar-se mais virulentos num ambiente hiperglicêmico. ${ }^{(3)}$

Um ensaio clínico randomizado publicado em 2001 ${ }^{(4)}$ demonstrou que, um controle glicêmico rígido em pacientes cirúrgicos criticamente doentes, reduziu a mortalidade hospitalar. A extrapolação destes dados a pacientes sépticos incorporou esta terapêutica as orientaçóes de tratamento destes pacientes. ${ }^{(5,6)}$ No entanto, ensaios clínicos randomizados posteriores $^{(7-9)}$ falharam em demonstrar o mesmo benefício aos pacientes críticos em UTIs cirúrgicas e clínicas.

Controvérsias vêm surgindo, portanto, em relação a uma conduta já estabelecida e aplicada mundialmente em ambiente de UTI. Diante disso, o objetivo desse trabalho é traçar um perfil dos pacientes que necessitam de controle glicêmico rigoroso em ambiente de UTI avaliando sua mortalidade a curto (durante a internação na UTI) e a longo prazo (após 2 anos da alta da UTI).

\section{MÉTODOS}

O presente estudo é uma coorte retrospectiva, em que foram acompanhados todos os pacientes que internaram em uma UTI clínico-cirúrgica de 18 leitos de um hospital universitário, no período de janeiro de 2007 até janeiro de 2008. O estudo teve a aprovação do Comitê de Ética da Instituição.

Foram analisadas as seguintes variáveis: idade, sexo, comorbidades associadas, motivo de internação da UTI (presença ou não de sepse), escores de gravidade como escore Acute Physiology and Chronic Health Evaluation (APACHE II), escala de coma de Glasgow, escore Sequential Organ Failure Assessment (SOFA), graduação da necessidade de suporte intensivo [escore Therapeutic Intervention Scoring System (TISS) calculado nas primeiras $24 \mathrm{~h}$ de internação (TISS 24h), tempo de internação na UTI, necessidade e tempo de ventilação mecânica (VM), insuficiência renal aguda (IRA) com ou sem necessidade de suporte dialítico. Além disso, foram avaliados desfechos clinicamente relevantes como infarto agudo do miocárdio (IAM), acidente vascular encefálico (AVC), polineuropatia da doença crítica (PNDC), úlcera de decúbito e mortalidade na UTI. Após a alta da UTI, os pesquisadores acompanharam a evolução hospitalar dos pacientes e realizaram contato telefônico com o paciente ou familiar mais próximo ( 2 anos após a alta da UTI) a fim de definir sobrevida ou morte do paciente. O Termo de Consentimento Livre e Esclarecido (TCLE) foi enviado via correio para a casa dos pacientes, juntamente com um envelope selado para facilitar a devolução do mesmo.

Para avaliação dos dados, os pacientes foram classificados em 2 grupos: uso de terapia insulínica contínua para controle glicêmico indicada pela equipe da UTI (Grupo TIC) e grupo de pacientes em que a terapia insulínica contínua não foi utilizada (Grupo NTIC).

\section{Análise estatística}

Os dados foram expressos em média \pm desvio-padrão (DP) ou percentagem de grupo. As variáveis categóricas foram analisadas com teste do Qui-quadrado e teste de Fisher; e as variáveis numéricas, com teste $t$-student para amostras independentes. Um $p<0,05$ foi considerado significativo. Uma análise multivariada foi realizada por regressão logística das variáveis que aumentaram o risco de morte pela avaliação univariada. Riscos relativos (RR) com intervalos de confiança de 95\% (IC95\%) foram calculados para estas. Os dados foram analisados pelo pacote SPSS versão 16.0. (Statistical Package for Social Science, Inc., Chicago IL, USA).

\section{RESULTADOS}

Dos 603 pacientes internados na UTI durante os 12 meses do estudo, $102(16,9 \%)$ receberam protocolo de insulina contínua visando controle glicêmico rigoroso (glicemia capilar $<150 \mathrm{mg} / \mathrm{dL}$ ), enquanto que os demais pacientes (n $=501 ; 81,3 \%)$ não necessitaram de tal controle.

A tabela 1 mostra a característica dos pacientes. A média de idade foi semelhante entre os grupos $(61,3 \pm 16,8$ anos vs. $61 \pm 17,6$ anos), bem como o gênero masculino (49\% versus $53,4 \%)$ e a prevalência de co-morbidades dos pacientes. Somente o grupo de diabéticos foi mais prevalente no grupo TIC $(34,3 \%$ versus $13,5 \%$; $p<0,001)$. Os pacientes que necessitaram TIC eram mais graves que os do grupo NTIC, escore APACHE II $(14 \pm 3$ versus $11 \pm 4 ; p=0,04)$, escore SOFA $(4,9 \pm 3,2$ versus $3,5 \pm 3,4 ; \mathrm{p}<0,001)$, escala de coma de Glasgow $(11,7 \pm 4,5$ versus $12,7 \pm 4,2 ; \mathrm{p}=0,03)$ e TISS nas primeiras $24 \mathrm{~h}(25,7 \pm 6,9$ versus $21,1 \pm 7,2 ; \mathrm{p}<0,001)$.

Quanto aos motivos de internação na UTI, foi destacada a presença ou não de sepse e de choque séptico. De todos os casos, 330 pacientes internaram por sepse, sendo 234 (70,9\%) com diagnóstico de choque séptico. Destes, 70 casos fizeram uso de protocolo insulínico e 164 não o fizeram. Os casos de choque séptico perfizeram $68,6 \%$ dos pacientes do grupo TIC e somente $32,7 \%$ dos pacientes do grupo NTIC.

As tabelas 2 e 3 demonstram que os pacientes do grupo TIC tiveram pior prognóstico durante a internação na UTI, quando comparados ao grupo NTIC. Houve maior necessidade de suporte dialítico $(34,3 \%$ versus $11,8 \%$; 
$\mathrm{p}<0,001)$, maior incidência de PNDC $(16,7 \%$ versus 5,6\%; $\mathrm{p}<0,001)$, desenvolvimento de úlceras de decúbito $(35,3 \%$ versus $8,8 \% ; \mathrm{p}<0,001)$ e maior necessidade de $\operatorname{VM}(88,2 \%$ versus $51,5 \% ; \mathrm{p}<0,001)$. A tabela 4 representa a análise multivariada das variáveis que, na análise univariada, sugeriram aumento de mortalidade dos pacientes. A necessidade de terapia insulínica apresentou RR para morte de 1,56 (IC95\% 1,02-1,87), porém muito inferior ao risco relativo da internação na UTI por choque séptico, 4,77 (IC95\% 3,37-7,43), necessidade de suporte ventilatório, 1,68 (IC95\% 1,42-2,11) e necessidade de suporte dialítico, 8,76 (IC95\% 6,39-10,32).

\section{Tabela 1 - Características dos pacientes}

\begin{tabular}{|c|c|c|c|}
\hline \multirow[t]{2}{*}{ Variáveis } & \multicolumn{3}{|c|}{ Controle glicêmico rigoroso } \\
\hline & $\operatorname{Sim}(N=102)$ & Não $(\mathrm{N}=501)$ & Valor de $\mathrm{p}$ \\
\hline Idade, anos & $61,3 \pm 16,8$ & $61 \pm 17,6$ & 0,86 \\
\hline Sexo masculino $(\mathrm{N}=318)$ & $50(49)$ & $268(53,4)$ & 0,48 \\
\hline \multicolumn{4}{|l|}{ Escores de gravidade } \\
\hline APACHE II & $14 \pm 3$ & $11 \pm 4$ & 0,04 \\
\hline Escala de Glasgow & $11,7 \pm 4,5$ & $12,7 \pm 4,2$ & 0,03 \\
\hline SOFA & $4,9 \pm 3,2$ & $3,5 \pm 3,4$ & $<0,001$ \\
\hline TISS - 24h & $25,7 \pm 6,9$ & $21,1 \pm 7,2$ & $<0,001$ \\
\hline \multicolumn{4}{|l|}{ Comorbidades associadas } \\
\hline Cardiopatia isquêmica $(\mathrm{N}=221)$ & $45(44,1)$ & $176(35,1)$ & 0,06 \\
\hline Hipertensão arterial sistêmica (234) & $46(45)$ & $189(37,7)$ & 0,07 \\
\hline Acidente vascular encefálico $(\mathrm{N}=68)$ & $10(9,8)$ & $58(11,6)$ & 0,73 \\
\hline Neoplasia $(\mathrm{N}=130)$ & $22(21,6)$ & $108(21,5)$ & 1,00 \\
\hline SIDA $(N=26)$ & $2(2)$ & $24(4,8)$ & 0,31 \\
\hline Diabete mélito $(\mathrm{N}=103)$ & $35(34,3)$ & $68(13,5)$ & $<0,001$ \\
\hline
\end{tabular}

APACHE - Acute Physiology and Chronic Health Evaluation; SOFA - Sequential Organ Failure Assessment; TISS - Therapeutic Intervention Scoring System; SIDA - Síndrome da Imunodeficiência Adquirida. Resultados expressos em N(\%), média \pm desvio padrão.

Tabela 2 - Desfechos clínicos e mortalidade

\begin{tabular}{lccc}
\hline Variáveis & Controle glicêmico rigoroso \\
Não $(\mathrm{N}=501)$ & Valor de $\mathrm{p}$ \\
\hline Desfechos clínicos & & & \\
IAM $(\mathrm{N}=16)$ & $1(1)$ & $15(3)$ & 0,49 \\
Acidente vascular encefálico $(\mathrm{N}=21)$ & $6(5,9)$ & $15(3)$ & 0,14 \\
Insuficiência renal aguda $(\mathrm{N}=145)$ & $52(51)$ & $93(18,5)$ & $<0,001$ \\
Polineuropatia $(\mathrm{N}=45)$ & $17(16,7)$ & $28(5,6)$ & $<0,001$ \\
Úlcera de decúbito $(\mathrm{N}=80)$ & $36(35,3)$ & $44(8,8)$ & $<0,001$ \\
Mortalidade $(\mathrm{N}=151)$ & $62(60,7)$ & $89(17,7)$ & $<0,001$ \\
\hline
\end{tabular}

IAM - infarto agudo do miocárdio. Resultados expressos em $\mathrm{N}(\%)$.

Tabela 3 - Desfechos intermediários relacionados a internaçáo na unidade de terapia intensiva

\begin{tabular}{lccc}
\hline Variáveis & Controle glicêmico rigoroso & \\
& Sim $(\mathrm{N}=102)$ & Não $(\mathrm{N}=501)$ & Valor de $\mathrm{p}$ \\
\hline Internação na UTI por sepse $(\mathrm{N}=330)$ & $78(76,5)$ & $252(50,2)$ & $<0,001$ \\
Presença de choque séptico $(\mathrm{N}=234)$ & $70(68,6)$ & $164(32,7)$ & $<0,001$ \\
Dias em UTI & $19 \pm 21$ & $9 \pm 20$ & $<0,001$ \\
Necessidade de ventilação mecânica $(\mathrm{N}=347)$ & $90(88,2)$ & $258(51,5)$ & $<0,001$ \\
Dias em ventilação mecânica & $13,8 \pm 14,5$ & $8,7 \pm 11$ & 0,001 \\
Necessidade de suporte dialítico $(\mathrm{N}=94)$ & $35(34,3)$ & $59(11,8)$ & $<0,001$ \\
Necessidade de suporte intensivo TISS - 72h & $26,1 \pm 7$ & $20,9 \pm 7,3$ & $<0,001$ \\
\hline
\end{tabular}

UTI - Unidade de terapia intensivo; TISS - Therapeutic Intervention Scoring System. Resultados expressos em N(\%), média \pm desvio padrão. 
Tabela 4 - Análise multivariada dos fatores relacionados a aumento da mortalidade

\begin{tabular}{lccc}
\hline Variáveis & Análise univariada & Análise multivariada & RR (IC95\%) \\
\hline APACHE II & 0,02 & 0,09 & $1,23(0,86-1,54)$ \\
Escala de Glasgow & 0,04 & 0,1 & $1,04(0,54-2,34)$ \\
SOFA & $<0,001$ & 0,01 & $3,22(1,23-5,64)$ \\
TISS - 24h & $<0,001$ & 0,01 & $2,99(1,98-4,06)$ \\
Internaçấo por choque séptico & $<0,001$ & $<0,01$ & $4,77(3,37-7,43)$ \\
Necessidade de ventilaçáo mecânica & 0,01 & 0,02 & $1,68(1,42-2,11)$ \\
Necessidade de suporte dialítico & $<0,001$ & $<0,001$ & $8,76(6,39-10,32)$ \\
Uso de terapia insulínica contínua & $<0,001$ & 0,03 & $1,56(1,02-1,87)$ \\
\hline
\end{tabular}

RR - risco relativo; APACHE - Acute Physiology and Chronic Health Evaluation; SOFA - Sequential Organ Failure Assessment; TISS - Therapeutic Intervention Scoring System.

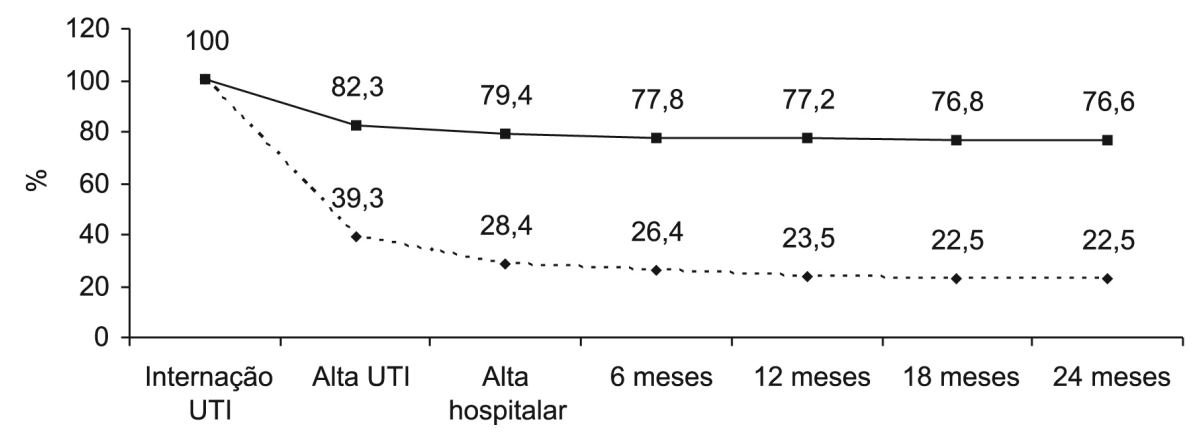

\begin{tabular}{lcccccccc}
\multicolumn{10}{c}{$\cdots \cdots \cdots$ TIC —-NTIC } \\
\hline Número de sobreviventes & Internação UTI & Alta UTI & Alta hospitalar & $6 \mathrm{~m}$ & $12 \mathrm{~m}$ & $18 \mathrm{~m}$ & $24 \mathrm{~m}$ \\
\hline TIC & 102 & 40 & 29 & 27 & 24 & 23 & 23 \\
NTIC & 501 & 412 & 398 & 390 & 387 & 385 & 384 \\
\hline
\end{tabular}

UTI - Unidade de terapia intensiva; TIC - uso de terapia insulínica contínua para controle glicêmico; NTIC - não uso de terapia insulínica; m - meses Resultados expressos em percentagem de pacientes, no gráfico e número de pacientes, na tabela.

Figura 1 - Curva de sobrevida dos pacientes os dois anos de acompanhamento dos grupos: TIC (uso de terapia insulínica contínua para controle glicêmico) e NTIC (não uso de terapia insulínica).

A figura 1 mostra a sobrevida dos pacientes durante os 2 anos de acompanhamento. A mortalidade total na UTI foi de $25 \%$ (151/603 pacientes) e maior no grupo TIC (60,7\% versus $17,7 \% ; \mathrm{p}<0,001)$. Esta diferença significativa se acentuou durante a internação hospitalar ( $71,6 \%$ versus $20,6 \%$; $\mathrm{p}<0,001)$ e nos primeiros 6 meses após a alta hospitalar $(73,6$ versus $22,2 \%$; $<<0,001)$, mantendo-se constante nos demais 18 meses de acompanhamento dos pacientes.

\section{DISCUSSÁO}

Esta coorte retrospectiva demonstrou que a necessidade de controle glicêmico rigoroso através do uso de protocolos de insulina contínua é um marcador de gravidade e de pior prognóstico dos pacientes internados na UTI, refletindo também maior mortalidade a longo prazo.

O controle glicêmico rigoroso, através do emprego de protocolos institucionais de insulina contínua, em pacientes criticamente doentes é adotado mundialmente e citado como recomendação em importantes Diretrizes, como o Surviving Sepsis Campaign de 2004, ${ }^{(5)}$ persistindo a recomendação na sua atualização de 2008. ${ }^{(6)}$ Também é referenciado por importantes associaçóes, como a Associação Americana de Endocrinologistas Clínicos ${ }^{(10)}$ e a Associação Americana de Diabetes. ${ }^{(11)}$ van den Berghe et al. ${ }^{(4)}$ demonstraram menor mortalidade nos pacientes randomizados para o grupo do controle glicêmico rigoroso entre 80 e $110 \mathrm{mg} / \mathrm{dl}$ (4,6\% versus $8 \%$; p $<0,04)$. No entanto, estudos posteriores, realizados em populaçôes mais heterogêneas (UTIs clínicas e clínico-cirúrgicas), não demonstraram o mesmo otimismo quanto à instituição desta terapêutica. Treggiari et al., ${ }^{(12)}$ incluindo 10.456 pacientes politraumatizados criticamente doentes e na sua maioria cirúrgicos, demonstraram uma tendência ao aumento da mortalidade na UTI (OR:1,15; IC95\%: 0,98-1,35) para o grupo de pacientes que receberam controle glicêmico rigoroso. van der Berghe et al., ${ }^{(7)}$ estudando pacientes predominan- 
temente clínicos, não evidenciaram redução da mortalidade hospitalar no grupo TIC (37,3\% versus $40 \%)$, exceto no subgrupo que permaneceu internado na UTI por 3 dias ou mais ( $43 \%$ versus $52,5 \%$; $p=0,009)$. Em nosso estudo, encontramos mortalidade na UTI maior no grupo TIC comparado com o grupo NTIC (60,7\% versus $17,7 \%, \mathrm{p}<0,001)$, com RR para morte de 1,56 (IC95\%: 1,02 - 1,87). Porém ressalta-se que tal achado está relacionado à gravidade dos pacientes no grupo TIC devido a maiores índices de APACHE II, SOFA, TISS-24h, TISS-72h e de maior internação na UTI por choque séptico, que demonstraram risco de morte superior ao da necessidade da terapia insulínica. Além disso, estes desenvolveram mais insuficiência renal aguda, polineuropatia do doente crítico e úlcera de decúbito, além de permanecerem mais tempo na UTI e mais tempo em VM, o que ratifica a gravidade dos mesmos. Em meta-análise publicada em $2008^{(13)}$ com 29 ensaios clínicos e $\mathrm{n}=8.432$, o controle glicêmico rigoroso foi associado à reduçáo significativa de sepse ,RR:0,76 (IC95\%: 0,59-0,97) , quando estudados pacientes cirúrgicos. Recente estudo ${ }^{(14)}$ demonstrou que, após 90 dias de randomização, o controle glicêmico rigoroso aumentou o risco absoluto de morte em 2,6 pontos percentuais, diferença mantida após ajuste de possíveis confundidores. Diante desses achados, os autores deixam a mensagem de que não recomendam o uso de um controle tão rigoroso quanto o já estabelecido pelas Diretrizes para pacientes criticamente doentes.

Diener et al. ${ }^{(15)}$ e Azevedo et al. ${ }^{(16)}$ não demonstraram diferença de mortalidade intra-hospitalar entre os grupos (TIC versus NTIC), nem diferenças em relação à recuperação neurológica após a alta hospitalar. Em nosso estudo não foram avaliadas seqüelas neurológicas após alta da UTI, somente taxas de mortalidade.

Previamente, dois estudos DIGAMI sequenciais, ${ }^{(17,18)}$ avaliando prognóstico a longo prazo, mostraram resultados diferentes. O primeiro mostrou que o controle glicêmico rigoroso diminui mortalidade em $29 \%$ ao final de 1 ano em pacientes admitidos no hospital com infarto agudo do miocárdio. Já o segundo, não confirmou tal achado numa população similar. Em nosso estudo, analisando dados após 2 anos da alta hospitalar e encontramos mortalidade maior no grupo TIC $(77,5 \%$ versus $23,4 \%$; $p<0,001)$. Portanto, a necessidade de controle glicêmico rigoroso através do uso de protocolos de insulina contínua durante a internação na UTI foi um marcador de aumento da mortalidade nos pacientes mesmo a longo prazo (2 anos após a alta da UTI).

Reiteramos que a presente coorte náo teve como objetivo avaliar se o controle glicêmico rigoroso influencia diretamente nos resultados, mas predizer a gravidade dos pacientes submetidos a esta intervenção e, principalmente, o prognóstico dos mesmos a longo prazo.

\section{CONTRIBUIÇÓES}

Márcia Inês Boff, Cassiano Teixeira e Cláudia da Rocha Cabral revisaram a literatura e escreveram o artigo. Roselaine Pinheiro de Oliveira, Márcio Pereira Hetzel e Daniele Munaretto Dallegrave coletaram os dados e contribuíram significativamente na construção do artigo. Cassiano Teixeira fez a análise estatística. Márcia Inês Boff garante a veracidade dos dados escritos.

\section{AGRADECIMENTOS}

Os autores agradecem ao staff técnico da UTI Central do Complexo Hospitalar da Santa Casa pela paciência e colaboração na coleta dos dados e execução do estudo.

\section{ABSTRACT}

Objectives: Stress-induced hyperglycemia is frequent in critically ill patients and has been associated with increased mortality and morbidity (both in diabetic and non-diabetic patients). This study objective was to evaluate the profile and long-term prognosis of critically ill patients undergoing tight glucose-control.

Methods: Prospective cohort. All patients admitted to the intensive care unit over 1 -year were enrolled. We analyzed demographic data, therapeutic intervention, and short- (during the stay) and long-term (2 years after discharge) mortality. The patients were categorized in 2 groups: tight glucose control and non-tight glucose-control, based on the unit staff decision.

Results: From the 603 enrolled patients, 102 (16.9\%) underwent tight control (glucose $<150 \mathrm{mg} / \mathrm{dL}$ ) while $501 \mathrm{pa}$ tients $(83.1 \%)$ non-tight control. Patients in the TGC-group were more severely ill than those in the non-tight control group [APACHE II score $(14 \pm 3$ versus $11 \pm 4, P=0.04)$, SOFA $(4.9 \pm 3.2$ versus $3.5 \pm 3.4, P<0.001)$ and TISS-24h $(25.7 \pm$ 6.9 versus $21.1 \pm 7.2, P<0.001)]$. The tight control group patients also had worse prognosis: [acute renal failure $(51 \%$ versus $18.5 \%, P<0.001)$, critical illness neuropathy $(16.7 \%$ versus $5.6 \%, P<0.001)]$ and increased mortality (during the ICU-stay $[60.7 \%$ versus $17.7 \%, P<0.001]$ and within 2 -years of the discharge [77.5\% versus $23.4 \% ; P<0.001]$ ).

Conclusion: Critically ill patients needing tight glucose control during the unit stay have more severe disease and have worse short and long-term prognosis.

Keywords: Blood glucose/analysis; Hyperglycemia/prevention \& control; Insulin/therapeutic use; Hypoglycemic agents/ therapeutic use; Prognosis; Intensive care 


\section{REFERÊNCIAS}

1. Yendamuri S, Fulda GJ, Tinkoff GH. Admission hyperglycemia as a prognostic indicator in trauma. J Trauma. 2003;55(1):33-8.

2. Langouche L, Vanhorebeek I, Vlasselaers D, Vander Perre $S$, Wouters PJ, Skogstrand K, et al. Intensive insulin therapy protects the endothelium of critically ill patients. J Clin Invest. 2005;115(8):2277-86.

3. Thompson MJ, Rossini AA, Mordes JP. Management in diabetes critically ill patients. In: Irwin RS, Rippe JM. Irwin and Rippe's intensive care medicine. 6th ed. Philadelphia: Lippincott Willians \& Wilkins; 2008. p. 124555.

4. van den Berghe G, Wouters P, Weekers F, Verwaest C, Bruyninckx F, Schetz M, et al. Intensive insulin therapy in the critically ill patients. N Engl J Med. 2001;345(19):135967.

5. Dellinger RP, Carlet JM, Masur H, Gerlach H, Calandra T, Cohen J, et al. Surviving Sepsis Campaign guidelines for management of severe sepsis and septic shock. Intensive Care Med. 2004;30(4):536-55.

6. Dellinger RP, Levy MM, Carlet JM, Bion J, Parker MM, Jaeschke R, et al. Surviving Sepsis Campaign: international guidelines for management of severe sepsis and septic shock: 2008. Intensive Care Med. 2008;34(1):17-60. Erratum in: Intensive Care Med. 2008;34(4):783-5.

7. van den Berghe G, Wilmer A, Hermans G, Meersseman W, Wouters PJ, Milants I, et al. Intensive insulin therapy in the medical ICU. N Engl J Med. 2006;354(5):449-61.

8. Brunkhorst FM, Engel C, Bloos F, Meier-Hellmann A, Ragaller M, Weiler N, Moerer O, Gruendling M, Oppert M, Grond S, Olthoff D, Jaschinski U, John S, Rossaint R, Welte T, Schaefer M, Kern P, Kuhnt E, Kiehntopf M, Hartog C, Natanson C, Loeffler M, Reinhart K; German Competence Network Sepsis (SepNet). Intensive insulin therapy and pentastarch resuscitation in severe sepsis. N Engl J Med. 2008;358(2):125-39.

9. Devos P, Preiser JC, Melot C. Impact of tight glucose control by intensive insulin therapy on ICU mortality and the rate of hypoglycemia: final results of the glucontrol study [abstract ]. Intensive Care Med. 2007;33 Suppl 2:S189.
10. Rodbard HW, Blonde L, Braithwaite SS, Brett EM, Cobin RH, Handelsman Y, Hellman R, Jellinger PS, Jovanovic LG, Levy P, Mechanick JI, Zangeneh F; AACE Diabetes Mellitus Clinical Practice Guidelines Task Force. American Association of Clinical Endocrinologists medical guidelines for clinical practice for the management of diabetes mellitus. Endocr Pract. 2007;13 Suppl 1:1-68. Erratum in: Endocr Pract. 2008;14(6):802-3. Multiple author names added.

11. American Diabetes Association. Standards of Medical Care in Diabetes-2008. Diabetes Care. 2008;31(Suppl 1):S1254.

12. Treggiari MM, Karir V, Yanez ND, Weiss NS, Daniel S, Deem SA. Intensive insulin therapy and mortality in critically ill patients. Crit Care. 2008;12(1): R29.

13. Wiener RS, Wiener DC, Larson RJ. Benefits and risks of tight glucose control in critically ill adults: a metaanalysis. JAMA. 2008;300(8):933-44. Erratum in: JAMA. 2009;301(9):936.

14. NICE-SUGAR Study Investigators, Finfer S, Chittock DR, Su SY, Blair D, Foster D, Dhingra V, et al. Intensive versus conventional glucose control in critically ill patients. N Engl J Med. 2009;360(13):1283-97.

15. Diener JRC, Prazeres CEE, Rosa CM, Alberton UC. Avaliaçáo da efetividade e segurança do protocolo de infusáo de insulina de Yale para o controle glicêmico intensivo. Rev Bras Ter Intensiva. 2006;18(3):268-75.

16. Azevedo JRA, Lima ERM, Cossetti RJD, Azevedo RP. Intensive insulin therapy versus conventional glycemic control in patients with acute neurological injury: a prospective controlled trial. Arq Neuropsiquiatr. 2007;65(3B):733-8.

17. Malmberg K, Rydén L, Hamsten A, Herlitz J, Waldenström A, Wedel H. Effects of insulin treatment on causespecific one-year mortality and morbidity in diabetic patients with acute myocardial infarction. DIGAMI Study Group. Diabetes Insulin-Glucose in Acute Myocardial Infarction. Eur Heart J. 1996;17(9):1337-44.

18. Melbin LG, Malmberg K, Norhammar A, Wedel H, Rydén L; DIGAMI 2 Investigators. The impact of glucose lowering treatment on long-term prognosis in patients with type 2 diabetes and myocardial infarction: a report from the DIGAMI 2 trial. Eur Heart J. 2008;29(2):166-76. 